There exists a metric space $S$ in which Axioms 1, 3, 4, and 5' hold true, but such that $S$ is not completely separable. Hence it does not follow that a space $S$ in which Axioms 1, 3, 4, and 5' hold true is a subset of a plane, even if it is assumed that $S$ is metric.

Duke UnIVERsity

\title{
ANALOGS OF THE STEINER SURFACE AND THEIR DOUBLE CURVES*
}

\author{
BY A. R. WILLIAMS
}

The equations $x_{1}: x_{2}: x_{3}: x_{4}=x^{n}: y^{n}: z^{n}: w^{n}$, where $x, y, z, w$ are linear functions of three homogeneous parameters, represent a rational surface of order $n^{2}$. For $n=2$ we have the well known Steiner surface. The particular subject of this paper is the double curve of such a surface and its representation on the plane. A few general properties must first be mentioned.

We take in the plane the reference system $x=0, y=0, z=0$, and $x+y+z \equiv-w=0$. The diagonals of the quadrilateral are $x+y \equiv-z-w=0$, etc. The vertices of the diagonal triangle are $(1: 1:-1:-1),(1:-1: 1:-1),(1:-1:-1: 1)$, the fourth coordinate being $w$. Corresponding to the diagonals, the surface has 3 multiple right lines of order $n$, each meeting two opposite edges of the tetrahedron in points which correspond to a pair of opposite vertices of the quadrilateral. If $n$ is even, the multiple lines are concurrent at $(1: 1: 1: 1)$, which is a point of order $3(n-1)$ for the surface, corresponding to the vertices of the diagonal triangle and to certain pairs of imaginary points when $n>2$. If $n$ is odd, the multiple lines are not concurrent, but are coplanar, meeting two by two at 3 points corresponding to the vertices of the diagonal triangle. The intersection of two multiple lines is then a point of order $2 n-1$ for the surface. The class of the surface is always $3(n-1)^{2}$. The only pinch points are the 6 in which the multiple lines meet the edges of the tetrahedron. Each coordinate plane contains a single curve of order $n$, and is tangent to the surface along that curve, the order of contact being $n-1$. When $n$ is even the section by a plane through a multiple line meets it in one variable real point, and

\footnotetext{
* Presented to the Society, March 18, 1933.
} 
has at the node of the surface a point of order $2 n-3$ with one real tangent. When $n$ is odd the section by a plane through a multiple line has a point of order $n-1$ with imaginary tangents where the multiple line meets the other two. Of variable intersections with the multiple line two at most are real. In either case the plane determined by two multiple lines, or all three, contains in addition only isolated points of the surface.

The 3 double lines of the Steiner surface constitute its double curve. But for $n=3$ the order of the surface is 9 , and we must have a double curve of order 27 , since the genus of a plane section is 1 . The 3 triple lines account for only 9 . The plane representation indicates that the double curve, aside from the triple lines, consists of points isolated from the continuous portion of the surface and corresponding to pairs of conjugate imaginary points in the plane. To the section by the plane $x_{1}-x_{2}=0$ corresponds the plane curve $(y-x)(y-\omega x)\left(y-\omega^{2} x\right)=0$. On the imaginary lines we may take $1: \omega:-t \omega^{2}:(1+t) \omega^{2}$ and $1: \omega^{2}:-t \omega$ $:(1+t) \omega$, respectively, for $x: y: z: w$. These give $x_{1}: x_{2}: x_{3}: x_{4}$ $=1: 1:-t^{3}:(1+t)^{3}$, the parametric equations of a real plane cubic. For $t=\omega$ and $t=\omega^{2}$ the double cubic has the acnode $(1: 1:-1:-1)$, which is the intersection of the two triple lines that do not meet $x_{1}=x_{2}=0$. There are evidently 6 such double cubics. The two that lie in planes through opposite edges of the tetrahedron have the same acnode.

Turning now to the general case, since $y-t x$ corresponds to a rational plane curve of order $n$ in the plane $x_{2}-t^{n} x_{1}=0$, we infer that there may be $(n-1)(n-2) / 2$ double curves of order $n$ in planes through the opposite edge $x_{3}=x_{4}=0$. We shall find that this is so. But this is not enough. We have the relation $\begin{aligned} &\left(n^{2}-1\right)\left(n^{2}-2\right) / 2-(n-1)(n-2) / 2=3 n(n-1) / 2 \\ &+6 n(n-1)(n-2) / 2+n(n-1)(n-2)(n-3) / 2 .\end{aligned}$

The left side is the order of the double curve. The first term on the right is the equivalent of the three multiple lines; the second term is the portion just described; and the third term indicates that there are probably on the surface $(n-1)(n-2)(n-3) / 2$ skew double curves each of order $n$. We shall find this to be the fact. It will be convenient to begin with $n$ odd. Let $e^{1}, e^{2}, \cdots$, $e^{n-1}$ be the complex $n$th roots of unity, $e$ being the root of smallest amplitude. Consider the equations 


$$
1+r e^{i}=s e^{k}, 1+r e^{n-i}=s e^{n-k},
$$

where $r$ and $s$ are real. Either of these relations implies the other. The graphical interpretation is obvious. We readily obtain the relations $s / r=\left(e^{i}-e^{-i}\right) /\left(e^{k}-e^{-k}\right), r=-\left(e^{k}-e^{-k}\right) /\left(e^{k-i}-e^{-k+i}\right)$, $s=-\left(e^{i}-e^{-i}\right) /\left(e^{k-i}-e^{-k+i}\right)$, where $e^{-i}$ is written for $e^{n-i}$. There are $(n-1)(n-2) / 2$ pairs of equations (1). Now we have in the plane the pair of conjugate points

$$
\begin{aligned}
& x: y: z: w=1: t:(1+t) r e^{i}:-(1+t) s e^{k}, \\
& x: y: z: w=1: t:(1+t) r e^{-i}:-(1+t) s e^{-k},
\end{aligned}
$$

where $t$ is a parameter. They give

$$
x_{1}: x_{2}: x_{3}: x_{4}=1: t^{n}:(1+t)^{n} r^{n}:-(1+t)^{n} s^{n} .
$$

If we take for $t$ a fixed real number and use for $i$ and $k$ the $(n-1)(n-2) / 2$ possible arrangements we get the acnodes of the rational curve of order $n$ lying on the surface in the plane $x_{2}-t^{n} x_{1}=0$, whose image is $y-t x=0$. But if we take $t$ variable, in general real, we get a double curve of order $n$ on the surface in the plane $x_{4} / x_{3}+s^{n} / r^{n}=0$. Its parametric equations are (3). Its image in the plane is the line pair $s e^{k_{z}}+r e^{i} w=0$ and $s e^{-k_{z}}$ $+r e^{-i} w=0$, or

$$
s^{2} z^{2}+r s\left(e^{k-i}+e^{-k+i}\right) z w+r^{2} w^{2}=0 .
$$

If we set $t=-1$ in (3), we see that the double curve meets the edge $x_{3}=x_{4}=0$ where the latter is met by the multiple line. This double curve must itself be rational. Its nodes correspond to appropriate pairs of values of $t$ in (3). Evidently these pairs are complex numbers and the nodes of the double curve are all acnodes. They are easily found from (1). For if we put for $t$ in (3) the $r e^{i}$ and $r e^{-i}$ determined by the $(n-1)(n-2) / 2$ pairs of equations (1), we shall have $(1+t)^{n}$ real. Furthermore it is easily shown that each acnode of the curve (3) is at the same time an acnode of a double curve lying in a plane through the opposite edge $x_{1}=x_{2}=0$. In other words, any pair of lines of the set (4) taken with any pair of similar character through $x=y=0$ give 4 imaginary points conjugate in pairs and corresponding to the same point of the surface which is an acnode for both double curves. The ratio $s / r=-1$ in the $(n-1) / 2$ cases when $k+i=n$. Thus there are $(n-1) / 2$ double curves in the central plane 
$x_{3}-x_{4}=0$. Their images are $z^{2}-\left(e^{2 i}+e^{-2 i}\right) z w+w^{2}=0, \quad(i=1$, $2, \cdots,(n-1) / 2)$. Each plane double curve, therefore, has $(n-1) / 2$ collinear acnodes on the intersection of its plane with the central plane through the opposite edge. If $s / r$ is not -1 , its negative as well as its reciprocal occurs. Moreover, the multiple line in $x_{3}+x_{4}=0$ is equivalent to the $(n-1) / 2$ double curves in $x_{3}-x_{4}=0$. Thus the entire system of double curves in planes through an edge of the tetrahedron is symmetrically and harmonically placed with respect to the faces that intersect in that edge.

To find the skew double curves we use the equations

$$
1+t e^{i}+r e^{i}=s e^{k}, 1+t e^{-i}+r e^{-j}=s e^{-k}
$$

where $t, r$, and $s$ are real. Solving for $r$ and $s$ we find $r=A t+B$, $s=C t+D$, where $A=-\left(e^{k-i}-e^{-k+i}\right) /\left(e^{k-j}-e^{-k+j}\right)$ and $B, C, D$ are similar expressions having the same denominator as $A$. We then have as before

$$
\begin{aligned}
& x: y: z: w=1: t e^{i}:(A t+B) e^{j}:-(C t+D) e^{k}, \\
& x: y: z: w=1: t e^{-i}:(A t+B) e^{-j}:-(C t+D) e^{-k},
\end{aligned}
$$

which give

$$
x_{1}: x_{2}: x_{3}: x_{4}=1: t^{n}:(A t+B)^{n}:-(C t+D)^{n},
$$

the parametric equations of a skew curve of order $n$. To find its image we eliminate $t$ by use of any two of the equations (6). Thus we find the line pair

(8) $B x+A e^{-i} y-e^{-i_{z}}=0$ and $B x+A e^{i} y-e^{i z}=0$. The product of the left hand members has of course real coefficients. The imaginary lines have one real point in common which is found by solving (8), and which may be obtained by putting. $-e^{-i} B D /(A C)$ and $-e^{i} B D /(A C)$ for $t$ in the first and second of equations (6). These values put for $t$ in (7) give the same real point. Thus the skew double curve (7) has an acnode corresponding to the vertex of the imaginary line pair.

When $n$ is even the result is the same, but there are certain modifications which deserve mention. If $n$ is odd a number whose $n$th power is real must be the product of a real number and an $n$th root of unity. But if $n$ is even the $n$th power of any $2 n$th root of unity is real, that is, -1 . Therefore, in forming equa- 
tions (1) and (5) for $n$ even we must use the $2 n$th roots of unity. If $e$ is the complex root of smallest amplitude, we have $e^{1}, e^{2}, \cdots, e^{2 n-1}$, where $e^{n}=-1$. In the equations

$$
1+r e^{i}=s e^{k}, 1+r e^{2 n-i}=s e^{2 n-k},
$$

we may take $i$ and $k$ less than $n$ since $e^{n+i}=-e^{i}$. Further, if we replace each root in $\left(1^{\prime}\right)$ by its symmetric with respect to the imaginary axis, that is, replace $i$ by $n-i$ and $k$ by $n-k$, we merely change the signs of $r$ and $s$ and interchange the 2 imaginary lines which are the image of the plane double curve. Thus we have as before $(n-1)(n-2) / 2$ pairs. Equation (4) for the image of a double curve remains the same, and $x_{4} / x_{3}=\left(-s e^{k} /\left(r e^{i}\right)\right)^{n}$ as before. It is easily seen that now $s / r$ is always positive, and the minus sign has no effect since $n$ is even; but $\left(e^{k} / e^{i}\right)^{n}$ is negative if $i$ and $k$ are of unlike parity; that is, if one is even and the other is odd. If $e^{i}$ and $e^{k}$ are symmetric with respect to the imaginary axis they are of the same parity, $s / r$ becomes 1 , and the corresponding plane curve lies in the central plane $x_{3}-x_{4}=0$. There are $(n-2) / 2$ such curves. Each has one of its acnodes at the intersection of the multiple lines. There is a further distinction. For $n$ odd there are $(n-1) / 2$ double curves in a central plane and all the other plane double curves are in separate planes. Now if in $\left(1^{\prime}\right)$, the exponent $i$ being less than $n / 2$ and $k \neq n / 2$, we replace $k$ by $n-k$, the ratio $s / r$ and the parity will be unchanged, but the plane double curve obtained will be different. We have, therefore, $(n-2)(n-4) / 2$ planes which contain 2 double curves. The point where such a plane meets a multiple line is an acnode on both curves, and a fixed quadruple point on the section by any plane through the multiple line. Such planes come in pairs. First interchanging $i$ and $k$ in $\left(1^{\prime}\right)$, and then replacing $i$ by $n-i$, we get 2 curves of the same parity as the preceding, the ratio $s / r$ being replaced by its reciprocal. That is, the ratio $x_{4} / x_{3}$ in the one plane is the reciprocal of the like ratio in the other. If we put $n / 2$ for one of the exponents in $\left(1^{\prime}\right)$ and then interchange them, we get a pair of planes related as above, and each containing one double curve. There are $(n-2) / 2$ such pairs. Such a plane meets a multiple line in a point which is an acnode on the double curve and on the section by any plane through the multiple line. We may summarize 
the above thus: If a plane $x_{4}=R x_{3}$ contains 1 or 2 double curves, $R x_{4}=x_{3}$ contains the same number. The reciprocal of any ratio $x_{4} / x_{3}$ occurs, but never the negative. The planes that contain the double curves are symmetrically but not harmonically placed with respect to the faces of the tetrahedron. To find the skew double curves we follow the method of equations (5). If we replace the three roots involved by their symmetrics with respect to the imaginary axis we get nothing new. Thus there are $(n-1)(n-2)(n-3) / 2$ skew double curves.

In closing, the case $n=4$ may be mentioned since it is the first in which skew double curves occur. The planes $x_{4}+4 x_{3}=0$, $4 x_{4}+x_{3}=0$, and $x_{4}-x_{3}=0$, each contain a double quartic. Their images are $(z+w)^{2}+z^{2}=0,(z+w)^{2}+w^{2}=0$, and $z^{2}+w^{2}=0$. There are thus eighteen such curves, three through each edge. There are three skew double quartics whose images are the imaginary line pairs $(x-y)^{2}+(z-w)^{2}=0,(x-z)^{2}+(y-w)^{2}=0$, $(x-w)^{2}+(y-z)^{2}=0$. The real point of each pair is a vertex of the diagonal triangle and corresponds to the node of the surface. Therefore, each skew double curve has an acnode at the latter point. In fact one of them is given by $x_{1}: x_{2}: x_{3}: x_{4}=1: t^{4}$ : $-(t-1)^{4} / 4:-(t+1)^{4} / 4$, and for $t= \pm i$ the right side becomes $(1: 1: 1: 1)$. If one of these surfaces could be illuminated in a dark place we should see the double curves forming a thread-like pattern attached at certain points to the continuous portion.

The University of California 